\title{
Theme issue on EIST
}

\author{
Alexander Thayer
}

Published online: 8 November 2013

(C) Springer-Verlag London 2013

Over the past several years, new interfaces, software, and learning technologies, as well as the ubiquitous application of interactive technology in informal and formal learning environments, have given rise to an influx in new opportunities for learning, information sharing, and teaching. Members of the human computer interaction (HCI) community have collaborated with others from related fields such as educational technology and the learning sciences to share research, ideas, and perspectives during a series of workshops held at recent $\mathrm{CHI}$ conferences. These workshops have focused on topics of interest such as gestural input, mobile interaction, gaming, child-computer interaction (CCI), and technologies to support learners with special needs. This special issue of personal and ubiquitous computing on educational interfaces, software, and technology (EIST) is an outgrowth of the work of this multidisciplinary community and provides an overview of the ways that these technologies are playing out in classrooms, in homes, and in the world beyond the schoolhouse.

The special issue begins with an article by Clayphan, Kay, and Weinberger. These scholars describe ScriptStorm, a tabletop interface of their own creation that stimulates brainstorming among a group of people. Building on the existing literature regarding the different stages of brainstorming, Clayphan et al. describe how the ScriptStorm can help a group of people accumulate more ideas and then cluster those ideas into different groups. Additionally, this new system can help users reflect on their ideas and think more deeply about them. The key decisions in their interface design were to use different colors for different ideas, as well as how to place those ideas based on

A. Thayer $(\square)$

Seattle, WA, USA

e-mail: huevos@uw.edu their similarities. This system was carefully designed to ensure high levels of affordability and flexibility. The performance of the system was checked through a user study with 36 participants from 2 universities. The results of the study demonstrate that users find this system helpful and reveal that tabletop interfaces can successfully aid teams in their brainstorming activities.

Munteanu et al. describe a mobile interface they developed that can help adults in developed countries who have low literacy skills. One of the key features of their interface is an assistance technology that can help users by reading content in general, and text in particular, more loudly. The interface can also help users with definitions when needed by drawing upon an embedded dictionary and by supplying appropriate words when users are writing new content. Munteanu et al. also included in a feature in their interface called "practice" that helps users practice different words with proper pronunciations and spellings. Furthermore, they added a feedback system that encourages users to learn more while using the interface. After testing their interface with appropriate learners, the authors report that their interface can successfully enhance users' literacy performance. They also report relevant, interesting facts regarding how people with low literacy interact with such an interface.

Anthony, Brown, Tate, Nias, Brewer, and Irwin present their analysis of a touch interaction data set that includes nearly 70 adults and children ages 7 years and older. This data set also includes over 10,000 touch interactions and nearly 7,000 gestures. The analysis that Anthony et al. present reveals why it is sometimes difficult to understand the touches that children make as they interact with systems that support touch interaction, as well as how unintentional touches are made. Based on the patterns and prevalence of different types of touches, the authors provide a variety of 
useful design implications in this paper. These implications include suggestions for avoiding unintentional touches, limiting the screen size, and so on. This article is quite useful to developers who are working on educational applications and systems that rely on touch input.

Escobedo, Ibarra, Hernandez, Alvelais, and Tentori design and develop tools that can "think" for autistic children. In this exploratory work, the authors explain each of the design implications that resulted from their longitudinal participant observation study. Engaging both the students and the teachers in their study, the authors do an excellent job of depicting the real challenges that exist for designers in this area. Escobedo et al. tested their design intervention with autistic children and analyzed their performance based on the feedback of the knowledgeable teachers who were also included in the study. The resulting design implications can serve as foundational knowledge for future researchers in this field.

Verbert et al. analyzed different dashboards ranging from mobile phone screens to much larger displays and discuss relevant learnings, reflections, and sense-making activities. The issues they address in this article tackle a number of important HCI design challenges that exist for professionals who are developing educational interfaces. The authors point out how to evaluate the learning processes that occur as people use such interfaces across different kinds of displays and dashboards. They further define the kinds of data that might be helpful to collect in order to gauge system performance among users, as well as discuss how to collect those data without intervening in the users' natural tasks and activities. Starting from some very detailed system-level design questions, Verbert et al. also pose crucial questions that are very important in the context of HCI and interface design.

Shaer and Valdes present three interfaces that help users understand three ideas of biodesign. These interfaces are intended to help university students develop clearer ideas about these topics through interaction with reality based interfaces. One of the key contributions of this paper is to extend the notion of educational interfaces as companions to traditional learning practice in formal learning environments, as opposed to interfaces that attempt to replace such practice. This paper also asks important questions regarding design and development processes related to the creation of such interfaces for educated adult users, and the potential use of these interfaces as companions to the mainstream education system.

Finally, Radu engages in an interesting discussion about the role of augmented reality (AR) in the mainstream education system. Radu conducted a survey of related literature to outline the benefits and challenges of current $A R$ systems, and the results of this thorough review are presented here. Radu also addresses important issues in learning as they relate to AR technology, including longterm memory retention, physical involvement, motivation, and collaboration. This article clearly demonstrates how current AR systems are limited, and how such systems need to be improved in the future. These improvements can be considered as design recommendations for future AR system developers and researchers alike. 